\title{
Treatment of massive haemoptysis with microcoil embolization after en bloc double-lung transplantation with bronchial artery revascularization
}

\author{
J. Carlsen*, U.G. Svendsen*, F. Efsen**, G. Pettersson+
}

\begin{abstract}
Treatment of massive haemoptysis with microcoil embolization after en bloc doublelung transplantation with bronchial artery revascularization. J. Carlsen, U.G. Svendsen, F. Efsen, G. Pettersson. CERS Journals Ltd 1997.

ABSTRACT: Successful treatment of a severe haemoptysis with microcoil embolization in an en bloc double-lung transplanted patient is described.

A 53 year old woman with advanced bronchiolitis obliterans syndrome experienced severe haemoptysis 26 months after an en bloc double-lung transplantation with direct bronchial artery revascularization using the left internal mammary artery.

Bronchoscopy showed that the haemoptysis originated from the lingula. Only two months after the transplant, left internal mammary arteriograms revealed proliferation and enlargement of the bronchial arteries in the lingula. The early occurrence of the vascular malformation indicated a pre-existing bronchiectasis in the donor lung, possibly due to tobacco smoking. After uncomplicated microcoil embolization of the left internal mammary artery, the patient experienced no further episodes of haemoptysis.

Microcoil embolization can be used successfully to treat massive haemoptysis related to proliferated and enlarged bronchial arteries in transplanted lungs with bronchial artery revascularization.
\end{abstract}

Eur Respir J 1997; 10: 492-493.

\author{
*Dept of Medicine B, The Heart Centre \\ **Dept of Diagnostic Radiology, Cardio- \\ vascular Section, Centre for Clinical Imaging \\ and Engineering, ${ }^{+}$Dept of Cardiothoracic \\ Surgery, The Heart Centre, Rigshospitalet, \\ University of Copenhagen, Denmark. \\ Correspondence: J. Carlsen \\ Dept of Medicine B, 2142 \\ Rigshospitalet \\ Blegdamsvej 9 \\ DK-2100 Copenhagen \\ Denmark \\ Keywords: Bronchial revascularization \\ bronchiectasis \\ haemoptysis \\ lung transplantation
}

Received: May 81996

Accepted after revision September 281996
Direct bronchial artery revascularization in human lung transplantation was first introduced by COURAUD et al. [1] in 1992, to improve airway healing and reduce early and late complications, but has been considered timeconsuming and associated with an increased risk of perioperative bleeding [2].

At The National University Hospital, Rigshospitalet, Denmark, single- and double-lung as well as heart-lung transplantations with direct bronchial artery revascularization have been performed successfully in more than 50 patients between 1992 and 1995 [3]. In this paper, we report the successful treatment of a massive haemoptysis possibly due to pre-existing bronchiectasis in the donor lungs after an en bloc double-lung transplantation with direct bronchial artery revascularization.

\section{Case report}

A 53 year old woman received an en bloc doublelung transplantation in August 1992 on the basis of $\alpha-1$ antitrypsin deficiency and severe pulmonary emphysema. The transplantation included direct bronchial artery revascularization using the left internal mammary artery (LIMA). Two months after the transplantation, arteriography demonstrated successful revascularization of only the left bronchial artery, with very sparse collaterals to the right side and a network of tortuous vessels in the lingula area (fig. 1a).

The patient was initially treated with standard quadruple immunosuppressive therapy, including antithymocyte globulin (ATG) induction, cyclosporin, azathioprine and prednisolone, and later with standard triple maintenance therapy. After an uncomplicated early course, the patient developed a severe acute rejection episode (grade LA4c) [4] 2 weeks postoperatively. Following rejection treatment, lung function improved, but over the next 12 months the lung function again slowly deteriorated. Progressive obliterative bronchiolitis was diagnosed [4], and, 22 months after the first transplantation, the patient was retransplanted with a right-sided single lung without any attempt at revascularization. An episode of moderate acute rejection (grade LA3a) [4] occurred 2 weeks after the retransplantation.

The patient was readmitted 26 months after the first transplantation and 4 months after the right-sided retransplantation following an episode of massive life-threatening haemoptysis. Six weeks prior to admission, episodes with blood-tinged sputum commenced. On admission, physical examination revealed slight dyspnoea and a chest radiograph revealed no changes. At bronchoscopy, blood could be traced directly into the lingula (segments 4 and 5 of the left lung). An internal mammary arteriogram was performed and showed the previously observed extensive network of tortuous proliferated bronchial arteries to be the possible source of bleeding (fig. 1b). Embolization of the LIMA was performed after percutaneous transfemoral catheterization using the Seldinger technique (fig. 1c) [5]. Under fluoroscopic control, a 6F mammary catheter (Cordis IM; Kodew, The Netherlands) was introduced into the origin of the LIMA as a guiding catheter. Through this catheter, the tip of a 3F delivery catheter (Cook, Bjaeverstock, Denmark) was placed in the LIMA $10 \mathrm{~mm}$ from the anastomosis, and four platinum Hilal Microcoils ${ }^{\mathrm{TM}}$ (Cook) with synthetic fibres 

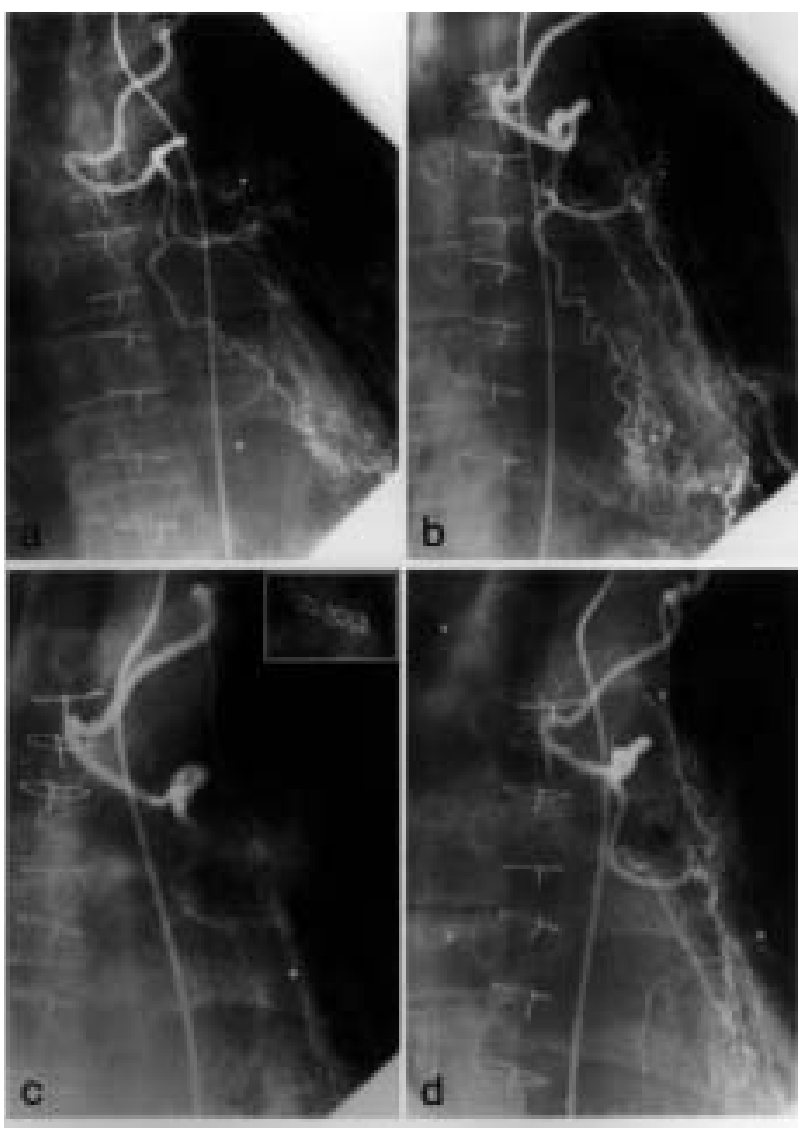

Fig. 1. - Postoperative internal mammary arteriograms. The recipient left internal mammary artery (LIMA) has been anastomosed to a left bronchial artery orifice in the donor descending thoracic aorta. ( $a$ and $b$ ). Filling of the bronchial arteries of the transplanted left lung from the LIMA graft. There was no direct filling of bronchial arteries to the right lung. Increased proliferation and enlargement of the bronchial arteries in the lingula is seen both 2 (a) and 26 (b) months after the double-lung transplantation. c) Arteriogram after embolization with platinum coils (insert), illustrating an incomplete occlusion of the LIMA 27 months after the initial transplantation. d) Arteriogram 3 months after embolization, showing the obstructed LIMA with very limited flow to the bronchial arteries.

were placed close to the anastomosis. At the final injection of contrast medium, the arterial flow was substantially reduced (fig. 1c).

Three months after the LIMA embolization, the patient was readmitted for control arteriography (fig. 1d). Although some reopening of the LIMA had occurred, there was still obstruction immediately after the coils, with very limited flow. Re-embolization was not attempted and no further episodes of haemoptysis occurred. The patient died of pulmonary insufficiency 5 months after the embolization, having severe bronchiolitis obliterans in the right lung also.

\section{Discussion}

Revascularization of the bronchial arteries at the time of lung transplantation has been performed with a high success rate, and may have resulted in improved airway healing as well as contributing to a better short- and medium-term survival [6-8]. One of our patients experienced an episode of severe haemoptysis 2 yrs after an en bloc double-lung transplantation, with successful direct bronchial artery revascularization of the left lung only. Four months previously, a single right lung retransplantation was performed due to advanced bronchiolitis obliterans, without any attempt at revascularization.

The important role of the bronchial arterial system in massive tracheobronchial bleeding is well-documented in inflammatory pulmonary diseases, including bronchiectasis [9]. Proliferation and enlargement of the bronchial arteries may occur in these diseases. Bronchial artery embolization is the treatment of choice of patients, with massive haemoptysis related to bronchiectasis [10].

In the present case, a series of arteriographies demonstrated an increased vascularity, with pathological bronchial arteries as early as 2 months after the primary double-lung transplantation, possibly due to bronchiectasis present in the donor lung. Although no clinical information of donor pulmonary disease was present at the time of transplantation, the changes might have been present in the donor lungs before transplantation. The donor was a 41 year old woman, who smoked 10-20 cigarettes a day. The episode of haemoptysis might then be aggravated by the bronchial artery revascularization.

In conclusion, this is the first report of successful treatment with microcoil embolization of massive haemoptysis related to proliferated and enlarged tortuous bronchial arteries (possibly due to pre-existing bronchiectasis in the donor lungs) after en bloc double lung-transplantation with direct bronchial artery revascularization. Bronchial artery revascularization and/or bronchiolitis obliterans syndrome were potential aggravating factors.

\section{References}

1. Couraud L, Baudet E, Martigne C, et al. Bronchial revascularization in double-lung transplantation: a series of 8 patients. Ann Thorac Surg 1992; 53: 88-94.

2. Patterson GA. Airway revascularization: Is it necessary? Ann Thorac Surg 1993: 56: 807-808.

3. Pettersson G, Nørgaard MA, Arendrup H, et al. En bloc double lung transplantation and direct bronchial artery revascularization surgical techniques and early outcome. J Heart Lung Transplant 1996; (in press).

4. Yousem SA, Berry GJ. Brunt EM, et al. A working formulation for the standardization of nomenclature in the diagnosis of heart and lung rejection: lung rejection study group. J Heart Transplant 1990; 9: 593-601.

5. Rémy J, Voisin G, Dupuis C, et al. Traitement des hémoptysies par embolisation de la circulation systémique. Ann Radiol 1974; 17: 5-16.

6. Daly RC, McGregor CGA. Routine immediate direct bronchial artery revascularization for single-lung transplantation. Ann Thorac Surg 1994; 57: 446-452.

7. Pettersson G, Arendrup H, Mortensen SA, et al. Early experience of double-lung transplantation with bronchial artery revascularization using mammary artery. Eur $J$ Cardiothorac Surg 1994; 8: 520-524.

8. McGregor CGA, Daly RC, Peters SG, et al. Evolving strategies in lung transplantation for emphysema. Ann Thorac Surg 1994; 57: 1513-1521.

9. Conlan AA. Massive hemoptysis - diagnostic and therapeutic complications. Surg Annu 1985; 17: 337-354.

10. Cremaschi P, Nascimbene C, Vitulo P, et al. Therapeutic embolization of bronchial artery: a successful treatment in 209 cases of relapse hemoptysis. Angiology 1993; 44: 295-299. 